\title{
Method of Formation of Quantitative Indicators of Complexity of the Environment by a Group of Autonomous Mobile Robots
}

\author{
Denis Beloglazov (D), Vladimir Pereverzev, Victor Soloviev (D), Viacheslav Pshikhopov, \\ and Morozov Roman
}

Research and Development Institute of Robotics and Control Systems, Southern Federal University, 105/42 Bolshaya Sadovaya Str., 344006 Rostov-on-Don, Russia

Correspondence should be addressed to Denis Beloglazov; d.beloglazov@gmail.com

Received 4 November 2019; Revised 17 January 2020; Accepted 3 February 2020; Published 30 April 2020

Academic Editor: Keigo Watanabe

Copyright (C) 2020 Denis Beloglazov et al. This is an open access article distributed under the Creative Commons Attribution License, which permits unrestricted use, distribution, and reproduction in any medium, provided the original work is properly cited.

\begin{abstract}
This article presents the methods that allow forming quantitative estimations of functional characteristics of a group of autonomous mobile robots (AMRs) and local areas of the environment surrounding it. The evaluation of the environment is based on the analysis of scattering of the cloud of obstacle points and made using the information formed by the computer vision system (CVS), which every robot in the group has. The core element of CSV is 3D lidar. Quantitative data about the complexity of the environment can be used for determining the optimal methods of solving the planning tasks and forming the patterns of group formation. Such data about the complexity of local areas of the environment can also be used for determining them as insurmountable for a single robot or a group of robots. The reason for such a decision can be the violation of safety rules and breaking of the group formation. The assessment of robots' functional characteristics is performed based on the fuzzy model that consists of separate fuzzy classifiers; each of them allows obtaining a quantitative estimation of one of the parameters. The hierarchic structure of the used fuzzy model makes it significantly easier to synthesize and analyze it.
\end{abstract}

\section{Introduction}

Robots are widely used in various spheres of human activity, such as the automotive industry, steel and chemical industry, and production of electronics. Further development of robotics is aimed at creating robots that are able to autonomously solve set problems and operate in groups. On the one hand, that makes robots less dependable on the human operator. On the other hand, that allows producing simple robots that operate in big and small mixed groups.

To make robots function within the environment, it is necessary to improve the methods of path planning, mapping, environment evaluation, and assessment of functional possibilities of single robots that make a part of the group [1-22]. In [1-6], methods of organizing an exchange and complexation of data that are formed by single members of the robot group when they solve a task of mapping the environment are proposed. A distinctive feature of the proposed methods is the possibility of their use with insufficient amount of sensory information. In $[7,8]$, methods for estimating the probability of successful completion of the task by robots are proposed, based on mathematical modeling of their interaction with each other and the surrounding space. The practical use of the proposed methods requires a big amount of a priori information about the parameters of robots and the environment. Such information cannot always be obtained in a sufficient amount and does not always have the desired level of precision, which significantly narrows the applicability of this article's findings.

In [9-11], methods of mobile autonomous mobile robot path planning for environments with dynamic obstacles are proposed. A distinctive feature of the methods is the ability to predict the behavior of dynamic obstacles, which is taken into account in the process of forming the trajectories of the movement of robots. 
In [12], a hybrid method of path planning for a group of mobile robots is considered. Its main idea is the consequent use of two methods-method of artificial potential fields and genetic algorithms (GA), and method of obtaining preliminary robots' path trajectory with its further optimization. A significant drawback of this method is the need for data on the location of obstacles, which is not always feasible in practice.

In $[13,14]$, methods of mobile robots' path planning based on classic GA are proposed. The peculiarity of the method described in [13] lies in the way of representing the generated trajectories by variable length chromosomes containing a set of pairs of values-the direction of movement and the length of the segment. The robot surrounding space is represented as the horizontal plane that is divided by sectors. That simplifies the process of genetic search but restricts the applicability of this method for the three-dimensional environment. The methods described in $[13,14]$, which have similar limitations, differ in the way of coding chromosomes.

In $[15,16]$, a method of mobile robots' path planning based on improved GA is proposed. Compared to analogs; the proposed algorithm has two significant differences: it uses data on the state of the environment that are limited by the visibility scope and applies a modified mutation operator. The main drawback of the methods described in $[15,16]$ is the same as of $[13,14]$ and connected with the representation of the environment.

In [17], a method was proposed for the formation of optimal trajectories for moving mobile robots based on a genetic algorithm similar to the methods proposed in [14-16]. Its distinctive feature is the use of a special polynomial to smooth the resulting GA trajectories.

In [18], different types of robot chassis' that are intended for increasing the off-road performance in the complex environment of both natural and artificial kinds are considered. Proposed decisions allow to significantly increase the area of use of mobile robots, but they are designed for the urban environment.

In [19], a method of reconfiguration of the structure of the snake-like wheel robot containing some base modules with the possibility to use each of them separately is considered. The main idea of the method is to minimize the time of base modules' reconfiguration by their more precise maneuvering.

In [20], a method of using a hive of robots for ground reconnaissance and mapping is proposed. It features the results of modeled hive landing on the surface of one of the planets of the Solar System, which confirm the efficiency of the method's algorithms of control and coordination.

In [21], a method for planning the path of moving mobile autonomous robots for three-dimensional environments, characterized by the possibility of optimizing the generated trajectories by using the particle swarm algorithm, is presented. The disadvantage of this method is the impossibility of its application in environment with dynamic obstacles.

In [22], a method is presented that allows one to obtain optimal robot trajectories based on an improved self-learning algorithm Q-learning. The disadvantages of the method are similar to those described in $[13,14]$ and are determined by the two-dimensional grid way of representing space. The common feature of most works on robotics, including [1-22], is the insufficient attention to the analysis of the environment in which robots perform the set tasks. Evaluation of robots' functional characteristics that allows determining the level of their fitness for the achievement of the set objectives is also not being done. In order to solve the above problems, this work presents the methods of formation of qualitative indicators of complexity of the local region of the environment and complex evaluation of robots' characteristics.

\section{Materials and Methods}

During the experiments with the proposed method, a number of the following assumptions were made: the relative position of the group members is known to each of its members; information on obstacles detected is available to all group members at the same time. In practice, the implementation of these assumptions requires the use of special equipment, such as inertial navigation systems, GPS navigation, odometers, and other devices to solve the problem of determining the location of robots in area, a data transmission system for organizing intragroup information exchange. Currently, the proposed method does not limit the source of data necessary for its correct application, which makes it possible to use any available hardware devices.

Under the "complexity of the environment" in this paper, we mean the number and configuration of obstacles in the environment surrounding the group of robots. Using data on the complexity of the environment and the parameters of the robots of the group, it is possible to obtain more optimal trajectories of movement of both individual members of the group and the entire group as a whole, to determine areas of space in which it is undesirable or unacceptable to enter.

The method of formation of qualitative indicators of complexity of the local region of the environment is based on the use of a particular model. It allows transforming data that come from the i-th robot's computer vision system into a numerical assessment of the local complexity of the environment $\delta_{l o c}$. Let us consider how this happens. When robots use CVS based on 3D lidars, the obstacles are presented as a set of points, which allows assessing their distribution. Figure 1 illustrates the scanning of the environment by CVS.

Using the physical analogy of the moment of rotation of the body around the longitudinal axis of an autonomous mobile robot (AMR) $O Y_{1}$ and the transverse axis $\mathrm{OY}_{3}$, and considering the body mass to be single, we obtain the concentration $J$ around the axes $\mathrm{OY}_{1}$ and $\mathrm{OY}_{3}$ for the point $P_{1}$ :

$$
\begin{aligned}
& J_{O Y p 1}=d_{p 11}^{2}, \\
& J_{O Y p 3}=d_{p 13}^{2} .
\end{aligned}
$$




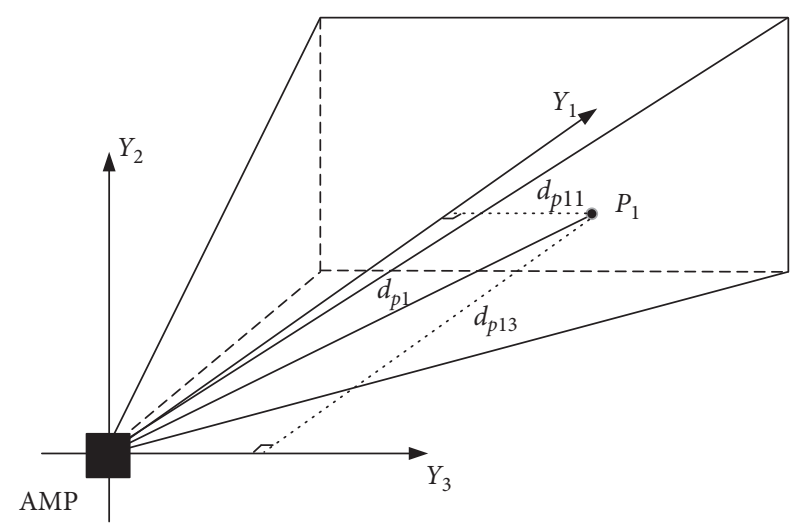

FIgURE 1: Illustration of CVS scanning the environment as a 3D lidar: $O Y_{1}-\mathrm{AMR}$ longitudinal axis; $\mathrm{O} Y_{2}-\mathrm{AMR}$ transverse axis; $\mathrm{P}_{1}$-obstacle point; $d_{p 11}$ and $d_{p 12}$-lengths of perpendiculars from the obstacle to the axes $\mathrm{O} Y_{1}$ and $\mathrm{O} Y_{3} ; d_{p 1}$-distance to the point $\mathrm{P}_{1}$.

Normalized concentrations of all the obstacle points around the axes $\mathrm{O} Y_{1}$ and $\mathrm{O} Y_{3}$ can be found by the following formulas:

$$
\begin{aligned}
& J_{O Y p 1}=\frac{\sum_{i=1}^{m} d_{p i 1}^{2}}{\sum_{j=1}^{m} d_{j 1}^{2}+\sum_{i=1}^{m} d_{p i 1}^{2}}, \\
& J_{O Y p 3}=\frac{\sum_{i=1}^{m} d_{p i 3}^{2}}{\sum_{j=1}^{m} d_{j 3}^{2}+\sum_{i=1}^{m} d_{p i 3}^{2}},
\end{aligned}
$$

where $d_{p i 1}$ and $d_{p i 3}$ are the lengths of perpendiculars from $i$ th obstacle to the axes $\mathrm{OY}_{1}$ and $\mathrm{OY}_{3}, d_{j 1}$ and $d_{j 3}$ are the lengths of perpendiculars from $j$-th lidar ray that did not meet an obstacle to the axes $\mathrm{OY}_{1}$ and $\mathrm{O} Y_{3}, m$ is the number of rays that met obstacles, and $n$ is the number of rays that did not meet an obstacle.

Let 3D lidar have the following characteristics: $\alpha_{\text {lid }}$ : horizontal scanning angle (rad); $\beta_{\text {lid: vertical scanning angle }}$ (rad); $l_{\text {lid }}$ lidar operating range (m); $\Delta_{\text {lid }}$ : lidar rays spacing (rad). Using the above characteristics, lidar is able to scan the space left-to-right and bottom-up. Lengths of perpendiculars $d_{p i 1}$ and $d_{p i 3}$ can be found by the following algorithm: calculate the horizontal angle of the $i$-th ray (left-to-right) $r h_{\mathrm{i}} \Delta\left(r h_{\mathrm{i}}\right.$ is the horizontal number of the $i$-th ray); calculate the vertical angle of the $i$-th ray (bottom-up) $r v_{\mathrm{i}} \Delta\left(r v_{\mathrm{i}}\right.$ is the vertical number of the $i$-th ray); calculate the coordinates $x_{i \mathrm{P}}$, $y_{i \mathrm{P}}$, and $z_{i \mathrm{P}}$ of the point $\mathrm{P}_{\mathrm{i}}$ using the formulas: $x_{i \mathrm{P}}=d_{p i} \mathrm{cos}$ $\left(r h_{i} \Delta\right) \times \cos \left(r v_{i} \Delta\right), y_{i \mathrm{P}}=d_{p i} \sin \left(r h_{i} \Delta\right) \times \cos \left(r v_{i} \Delta\right), z_{i \mathrm{P}}=d_{p i} \cos$ $\left(r h_{i} \Delta\right) \times \sin \left(r v_{i} \Delta\right)$; to take into account the AMR orientation angles $(\varphi, v, \gamma)$, we need to multiply the $\left(x_{i}, y_{i}, z_{i}\right)$ coordinate vector by rotation matrix:

$$
\begin{array}{r}
\mathrm{M}(\varphi, v, \gamma)=\left[\begin{array}{ccc}
c(\varphi) c(v) & c(\varphi) s(v) s(\gamma)-s(\varphi) c(\gamma) & c(\varphi) s(v) c(\gamma)+s(\varphi) s(\gamma) \\
s(\varphi) c(v) & s(\varphi) s(v) s(\gamma)+c(\varphi) c(\gamma) & s(\varphi) s(v) c(\gamma)-c(\varphi) s(\gamma) \\
-s(v) & c(v) s(\gamma) & c(v) c(\gamma)
\end{array}\right], \\
\delta_{\text {loc }}=\frac{J_{\mathrm{OY} 1}}{J_{\mathrm{OY} 3}}
\end{array}
$$

where $s(\cdot)$ and $c(\cdot)$ are sines and cosines, respectively; the direction cosines:

$$
\begin{aligned}
& \alpha=a \cos \left(\frac{x_{1 P}}{d_{p 1}}\right), \\
& \beta=a \cos \left(\frac{y_{1 P}}{d_{p 1}}\right), \\
& \rho=a \cos \left(\frac{z_{1 P}}{d_{p 1}}\right) .
\end{aligned}
$$

The lengths of perpendiculars from the point $P_{\mathrm{i}}$ to the axes $\mathrm{OY} Y_{1}$ and $\mathrm{OY} Y_{2}$ were found: $d_{p i 1}=d_{p i} \sin (\alpha)$ and $d_{p i 3}=d_{p i} \cos (\beta)$. Last expressions allow calculating the concentrations of the obstacle points using formulas (1)-(4).

To obtain the assessment of degree of the local complexity of the environment, we can use the following expression:
In order to confirm the adequacy of the assessment of the local complexity of the environment, we performed simulation with the following given data: 3D lidar parameters: $\alpha_{\text {lid }}=\pi / 2, \beta_{\text {lid }}=\pi / 4, l_{\text {lid }}=20 \mathrm{~m}, \Delta_{\text {lid }}=\pi / 30$, and the space is scanned left-to-right and bottom-up; moving object parameters: $y_{1}=0, y_{2}=0, y_{3}=0, \varphi=0, v=0$, and $\gamma=0$; and obstacle parameters: spheres with a unit radius.

Below, the examples of cases with different given number of obstacles in the view of 3D lidar and different given distance from AMR to the obstacles are given.

Case 1: there is one obstacle in the AMR's view at the distance of $15 \mathrm{~m}$. The vertical view projection for this case is given in Figure 2; the front view projection is shown in Figure 3.

In Case 1, we obtained the value of the local complexity of the environment of $\delta_{\text {loc }}=0.0115$, which corresponds to reality, since the obstacle is far from AMR. Case 2: there is an obstacle in the AMR's view at the distance of $5 \mathrm{~m}$, which is three times closer than that in Case 1. The vertical view projection for this case is presented in Figure 4, and the front 


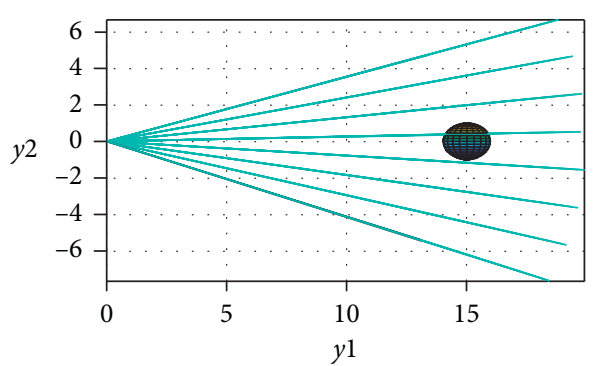

FIGURE 2: Vertical projection of the 3D lidar's view for one obstacle in $15 \mathrm{~m}$.

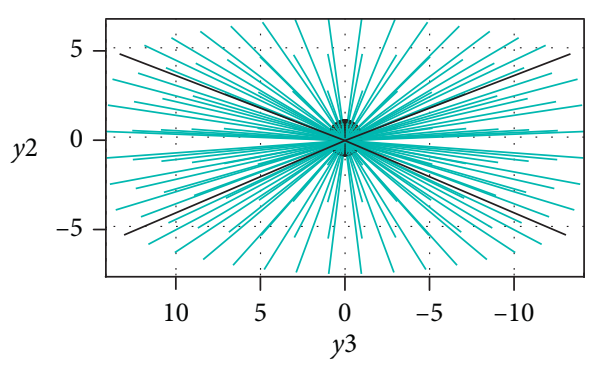

Figure 3: Front projection of the 3D lidar's view for one obstacle in $15 \mathrm{~m}$.

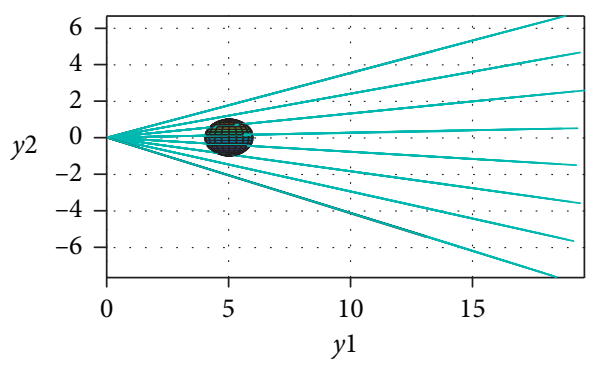

FIGURE 4: Vertical projection of the 3D lidar's view for one obstacle in $5 \mathrm{~m}$.

view projection is presented in Figure 5. In Case 2, we obtained the value of the local complexity of the environment of $\delta_{l o c}=0.0658$. The value of complexity estimation increased because the obstacle is closer to the AMR now. Case 3: there is one obstacle in the AMR's view at the distance of $1.35 \mathrm{~m}$. Figure 6 illustrates the tridimensional view of the obstacle as seen by the AMR's $3 \mathrm{D}$ lidar.

In Case 3, the value of the local complexity of the environment in the direction of AMR's movement is $\delta_{\text {loc }}=1$, which corresponds to reality, since the obstacle, in fact, blocks the way. Case 4: there are three relatively grouped obstacles in the AMR's view at the distance of $15 \mathrm{~m}$. The vertical view projection for this case is presented in Figure 7 , and the front view projection is presented in Figure 8. In Case 4 , we obtained the value of the local complexity of the environment of $\delta_{\text {loc }}=0.0483$. The obtained estimation value is bigger than that in Case 1-though the distance to the obstacles is the same, there are now three of them, which form a group. Case 5: there are three relatively grouped obstacles in the AMR's view at the distance of $5 \mathrm{~m}$.

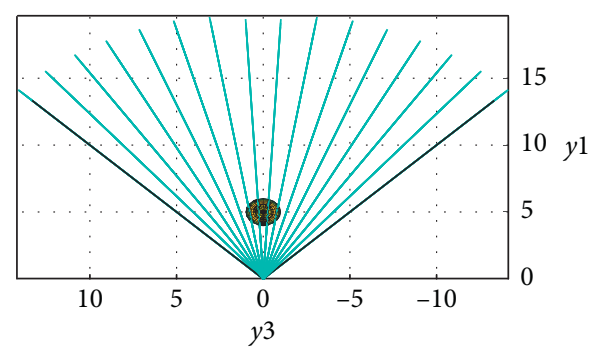

FIGURE 5: Front projection of the 3D lidar's view for one obstacle in $5 \mathrm{~m}$.

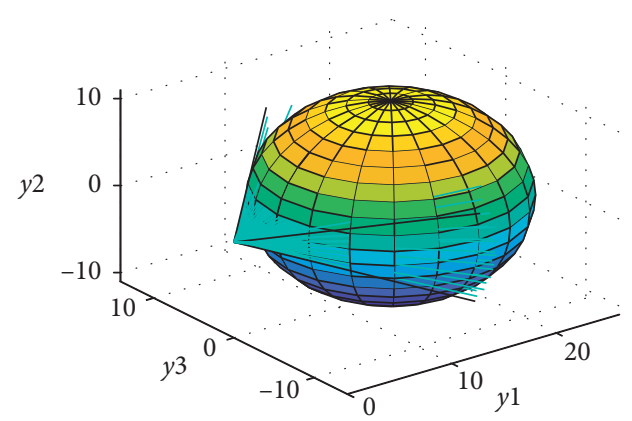

FIGURE 6: Tridimensional view of the obstacle at the distance of $1.35 \mathrm{~m}$ by the AMR's 3D lidar.

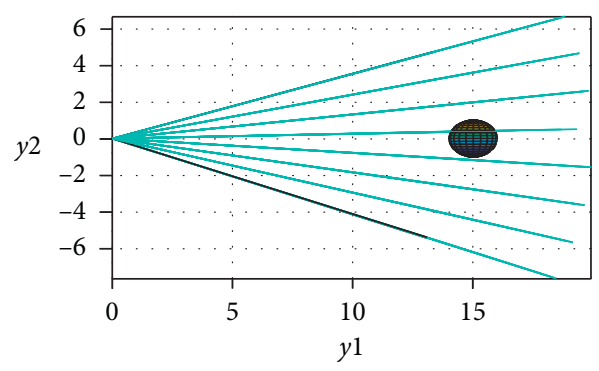

FIGURE 7: Vertical projection of the 3D lidar's view for three obstacles in $15 \mathrm{~m}$.

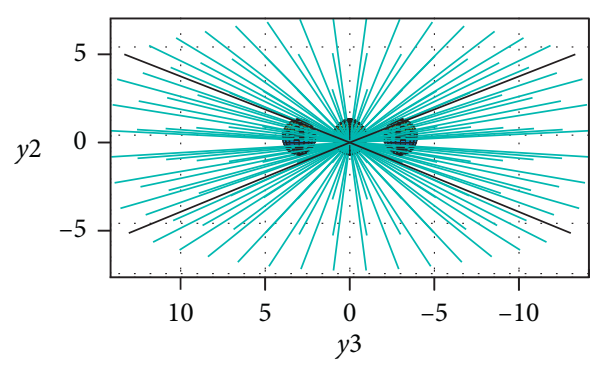

FIGURE 8: Front projection of the 3D lidar's view for three obstacles in $15 \mathrm{~m}$.

The vertical view projection for this case is presented in Figure 9, and the front view projection is presented in Figure 10. In Case 5, we obtained the value of the local complexity of the environment of $\delta_{l o c}=0.8153$. The value of the environment complexity estimation is now significantly bigger, comparing with the previous case that had the same 


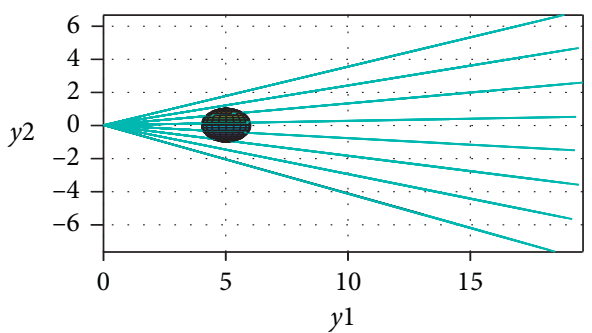

FIGURE 9: Vertical projection of the 3D lidar's view for three obstacles in $5 \mathrm{~m}$.

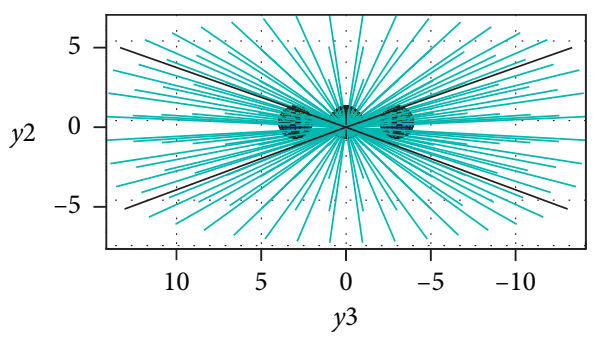

FIGURE 10: Front projection of the 3D lidar's view for three obstacles in $5 \mathrm{~m}$.

number of obstacles, but at three times bigger distance from the AMR. Case 6: there are five relatively grouped obstacles in the AMR's view at the distance of $15 \mathrm{~m}$. The vertical view projection for this case is presented in Figure 11, and the front view projection is presented in Figure 12.

In Case 6, we obtained the value of the local complexity of the environment of $\delta_{l o c}=0.0531$, which is bigger than that in Case 4 with three obstacles placed at the same distance. Case 7: there are five obstacles far from each other in the AMR's view at the distance of $15 \mathrm{~m}$. The vertical view projection for this case is presented in Figure 13, and the front view projection is presented in Figure 14. In Case 7, we obtained the value of the local complexity of the environment of $\delta_{l o c}=0.1784$, which is bigger than that in Case 4 with three obstacles and corresponds to the level of five obstacles in the AMR's 3D lidar view.

Successful completion of tasks by a group of robots is determined not only by the complexity of the environment but also by robots' characteristics. Obtaining a quantitative assessment of characteristics of members of an AMR group is a separate task, which is solved in this work basing on the model shown in Figure 15. This model consists of the following elements: model of assessment of the AMR's dynamics; model of assessment of the AMR's size; and model of assessment of the AMR's maneuvering capabilities. Decomposition of the model shown in Figure 15 into its constituent elements significantly reduces the complexity of the synthesis. This is achieved by reducing the number of combinations of input and output variables that the researcher is forced to operate when drawing up the control rules for each of the elements. Instead of one bulky fuzzy model, the result is a few simpler and logically transparent ones.

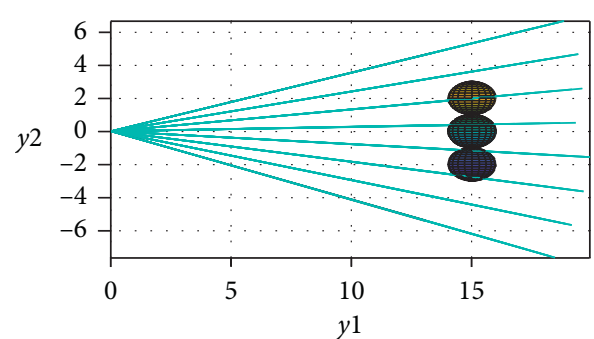

FIgURE 11: Vertical projection of the 3D lidar's view for five obstacles in $15 \mathrm{~m}$.

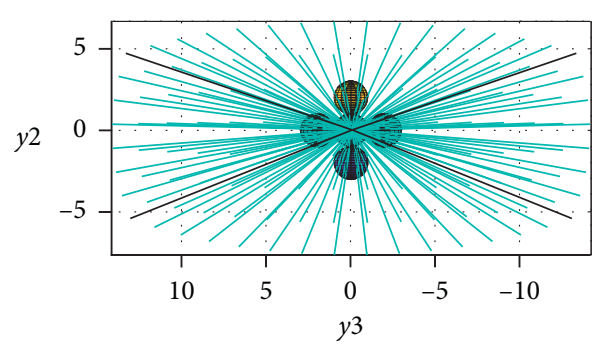

FIgURE 12: Front projection of the 3D lidar's view for five obstacles in $15 \mathrm{~m}$

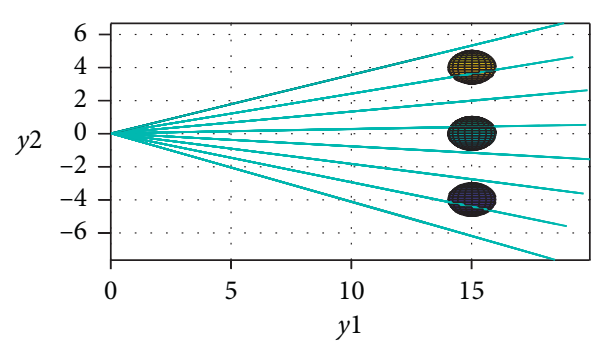

FIGURE 13: Vertical projection of the 3D lidar's view for five scattered obstacles in $15 \mathrm{~m}$.

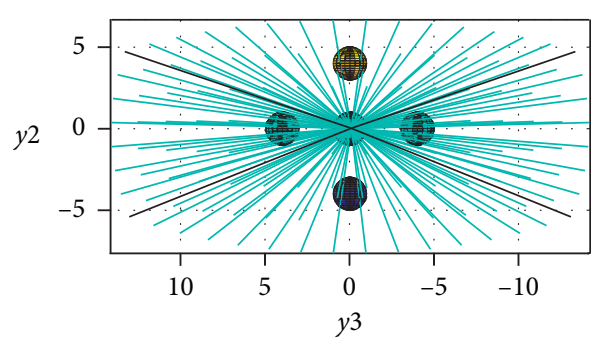

FIGURE 14: Front projection of the 3D lidar's view for five scattered obstacles in $15 \mathrm{~m}$.

We are going to present the model of assessment of the robot's dynamics as a combination of three models M1-M3 as follows: the input of the model receives output variables of fuzzy classifiers regarding the linear and angular characteristics of AMR, and the output variable $m_{r}$ is formed. Linear velocity $v_{r}$ and linear acceleration $a_{r}$ will be considered as the input linguistic variables (LV) of model M1. Angular velocity $\omega_{r}$ and angular acceleration $\varepsilon_{r}$ will be considered as the input linguistic variables of model M2. 


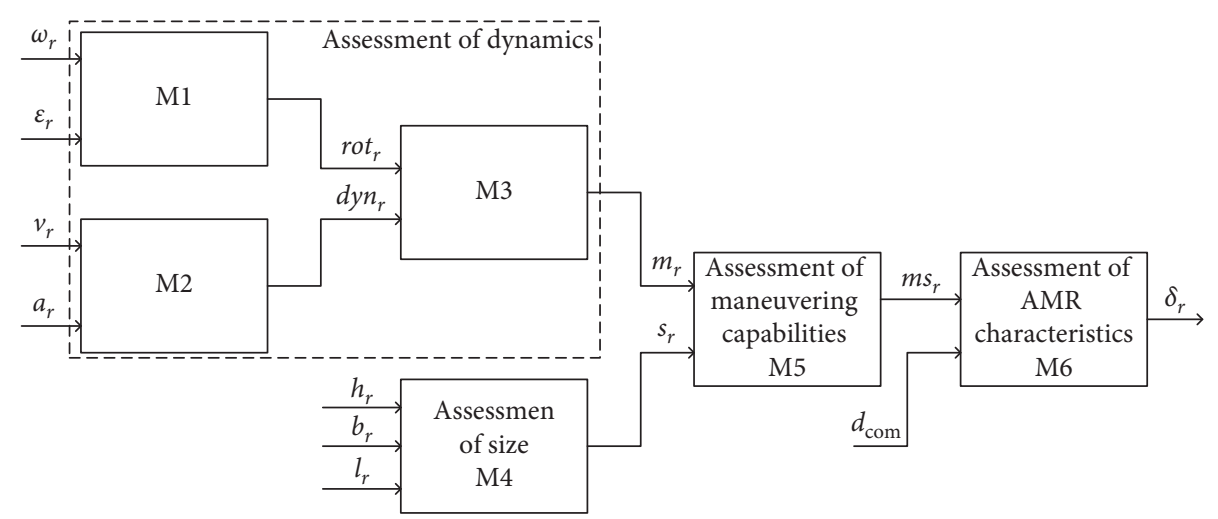

FIGURE 15: Structure of the model of assessment of the AMR's characteristics.

For the LV "linear velocity," experts determined the term set $T\left(v_{r}\right)=\{\langle$ low $\rangle,\langle$ medium $>,\langle$ high $\rangle$, which contains three terms-three fuzzy variables (FV). The base set for defying FV LV "linear velocity" has the velocity change range from $v_{\text {rmin }}$ to $v_{\text {rmax }}$. To simplify further operations with fuzzy values, let us normalize the range of velocity changes, reducing it to the interval $[0,1]$. Figure 16 shows the FV membership function for LV $v_{r}$. For the LV "linear acceleration," experts determined the term set $T\left(a_{r}\right)=\{\langle$ slow $\rangle$, $<$ average $>,<$ fast $>\}$, which contains three FVs. Note that the term "linear acceleration" includes both the acceleration and retardation of an AMR. The base set for setting FV LV "linear acceleration" has the acceleration change range from $-a_{\text {rmin }}$ to $+a_{\text {rmax }}$.

Again, we simplify further operations with fuzzy values; let us normalize the range of acceleration changes, reducing it to the interval $[0,1]$. Figure 17 shows the FV membership function for $\operatorname{LV} a_{r}$.

In the same way, let us define the LV $\varepsilon_{r}$ ("angular velocity") and LV $\omega_{r}$ ("angular acceleration"), setting the similar term sets $T\left(\varepsilon_{r}\right)$ and $T\left(\omega_{r}\right)$ with three FVs with the same meaning. Figure 18 shows the FV membership function for LV $\varepsilon_{r}$. Figure 19 shows the FV membership function for LV $\omega_{r}$. The base sets for determining FV for LV $\varepsilon_{r}$ and $\mathrm{FV}$ for $\mathrm{LV} \omega_{r}$ are also normalized, i.e., reduced to the range of values $[0,1]$. Rule bases of fuzzy classifiers $M 1$ and M2 contain 9 rules of fuzzy output each. Surfaces of fuzzy output for these models are shown in Figures 20(a) and 20(b). Output variable of the model of assessment of the AMR's dynamics is the one LV $m_{r}$ ("assessment of the AMR's dynamics").

This LV has the term set $T\left(m_{r}\right)=\{<$ low $\rangle,<$ medium $>$, $<$ high $>$ \}. The base set for assessment of the AMR's dynamics is the interval of relative values from 0 to 1 , where 1 is the maximum value of AMR's mobility. Figure 21 shows the FV membership function for LV $m_{r}$. Figure 22 shows the surface of fuzzy output of LV $m_{r}$ values, which illustrates the dependency of the degree of decision from the input parameters of the model.

Fuzzy model proposed for assessment of the AMR's size has three inputs and one output, as shown in Figure 22. For LV "length," experts determined the term set $T\left(l_{r}\right)=$ $\{<$ small $>$, $<$ medium $>$, $<$ great $>\}$, which contains three fuzzy

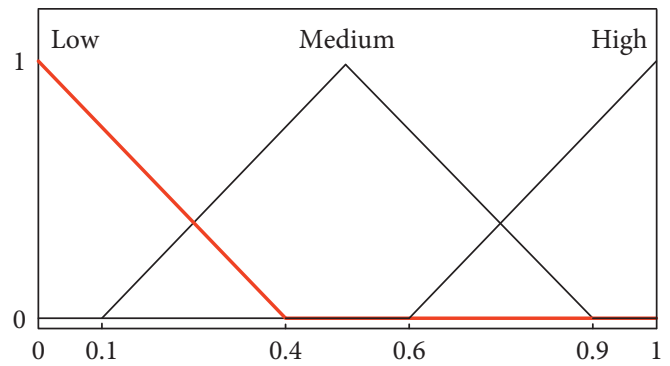

FIGURE 16: FV membership functions for LV $v_{r}$ : "linear velocity".

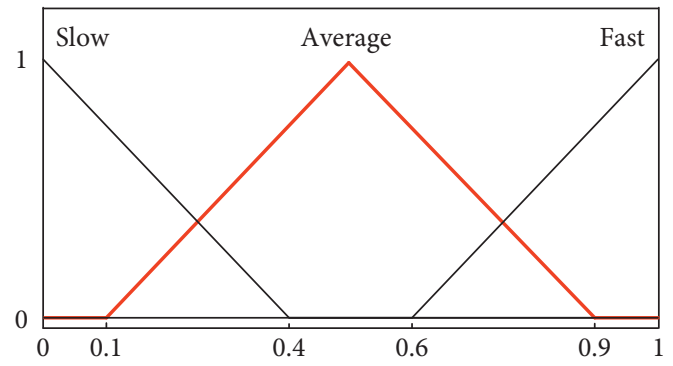

Figure 17: FV membership functions for LV $a_{r}$ : "linear acceleration".

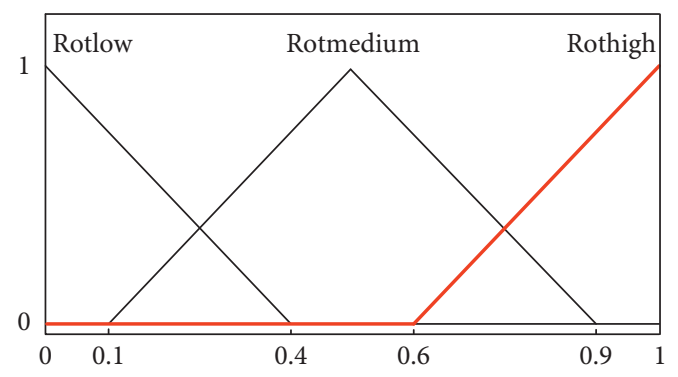

FIGURE 18: FV membership functions for LV $\varepsilon r$ : "angular velocity".

variables. The base set for determining FV LV "length" has the change range from $l_{\text {rmin }}$ to $l_{r \max }$, which is reduced to the interval $[0,1]$. Figure 23 shows the FV membership function for LV $l_{r}$. The LVs $h_{r}$ ("height") and и $b_{r}$ ("width") are defined in the same way. Membership functions of these variables are given in Figures 24(a) and 24(b). The output of 


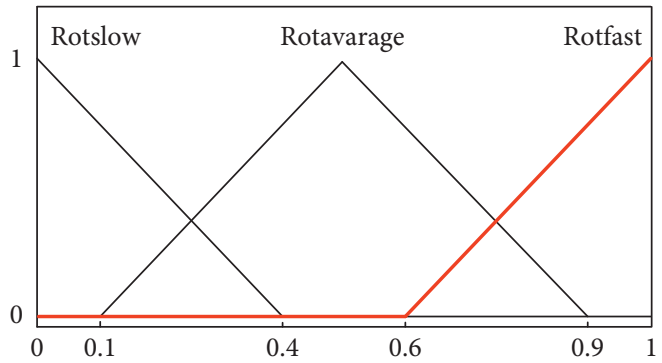

FIgURE 19: FV membership functions for LV $\omega_{r}$ : "angular acceleration".

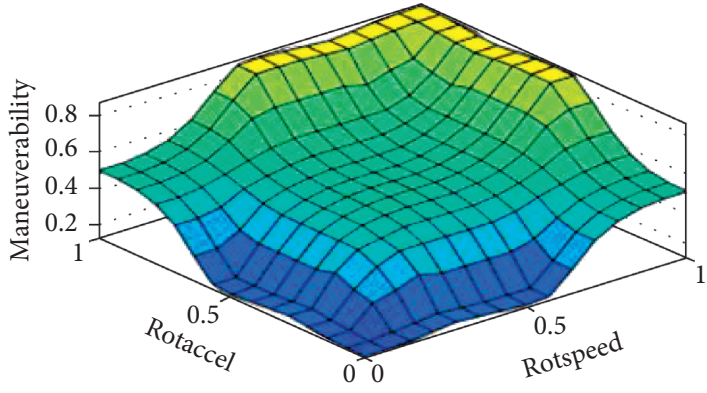

(a)

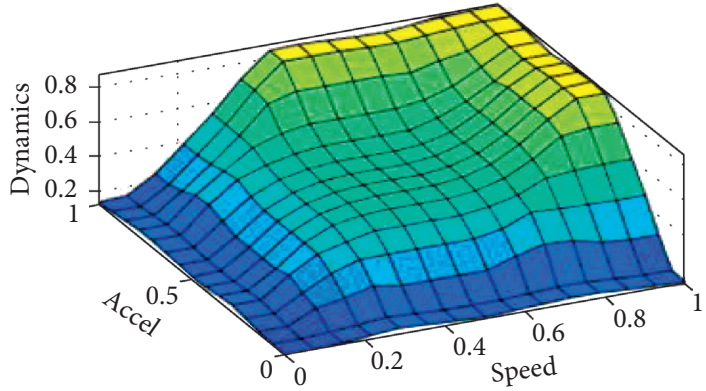

(b)

Figure 20: (a) Fuzzy output surfaces for model M1 and (b) fuzzy output surfaces for model M2.

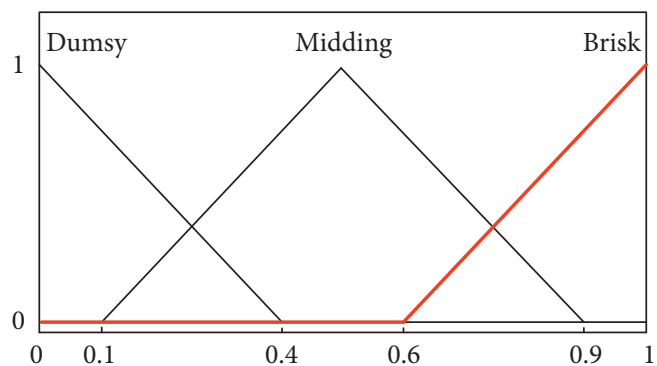

FIGURE 21: FV membership functions for LV $m_{r}$ : "AMR dynamics".

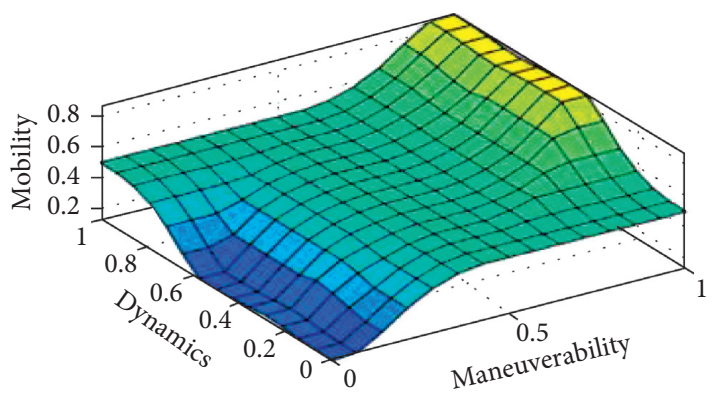

FIgURE 22: Fuzzy output surface for model M3.

the model of assessment of AMRs' size is defined by LV Size $r$ with term set $T\left(\right.$ Size $\left._{r}\right)=\{\langle$ small $>,<$ middle $\rangle,<$ large $\rangle$.

Figure 25 shows the FV membership function for LV Size $_{r}$ with base set of estimations from 0 to 1 . The expert rule base for determining the values of the variable Size $_{r}$ contains 27 rules. Fuzzy model of integral estimation of AMR's

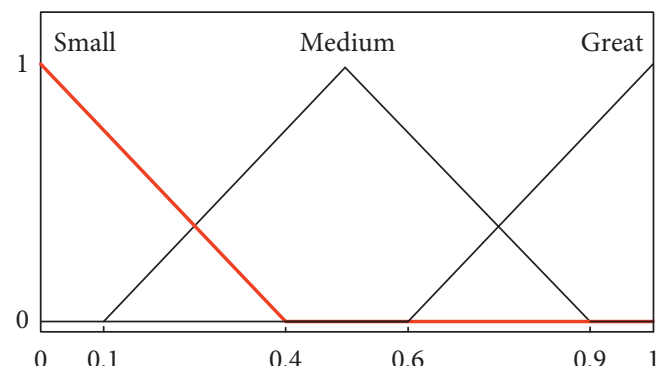

Figure 23: FV membership functions for LV $l_{r}$ : "length".

maneuvering capabilities has two inputs ("assessment of dynamics" and "assessment of size") and one output (linguistic variable "maneuvering capabilities"). For LV "maneuvering capabilities," experts determined the term set $T$ $\left(m s_{r}\right)=\{<$ weak $>,<$ strong $>,<$ excellent $>\}$, which contains three fuzzy variables. The base set for defying FV LV "maneuvering capabilities" has the changing range from $m s_{r \min }$ to $m s_{\text {rmax }}$, which is reduced to the interval $[0,1]$.

Figure 26 shows the FV membership function for LV $m s_{r}$. The form of membership functions for variable $d_{c o m}$ is shown in Figure 27. The rule base for output values of the variable "maneuvering capabilities" contains 9 rules. Figure 28 shows the fuzzy output surface for model M5.

\section{Results and Discussion}

The initial parameters of the fuzzy models used in the work were selected based on the preferences of the researchers and subsequently adjusted according to the results of experiments. The final parameters of fuzzy models, as well as the 


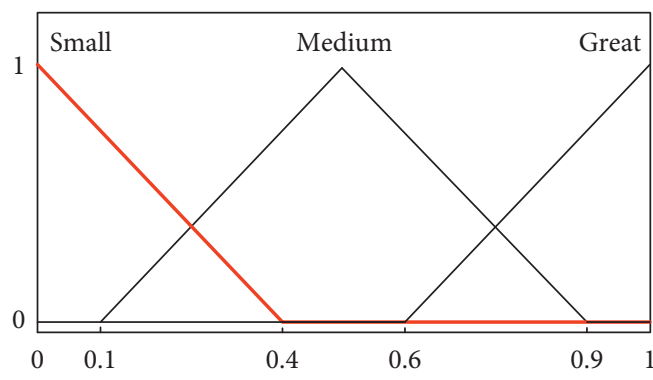

(a)

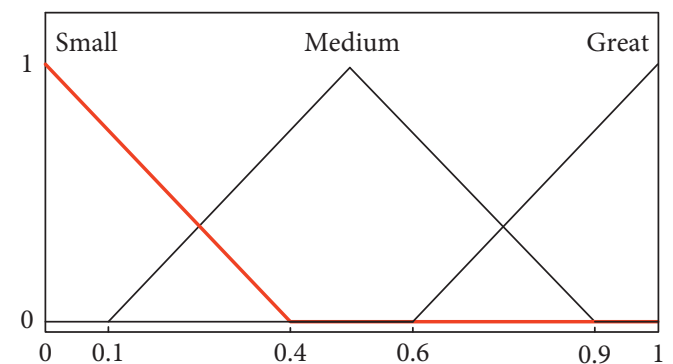

(b)

Figure 24: (a) FV membership functions for LV $h_{r}$ : "height"; (b) FV membership functions for LV $b_{r}$ : "width".

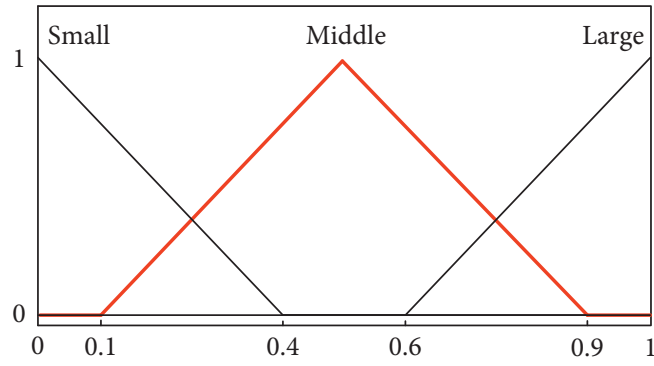

Figure 25: FV membership functions for LV Size $_{r}$.

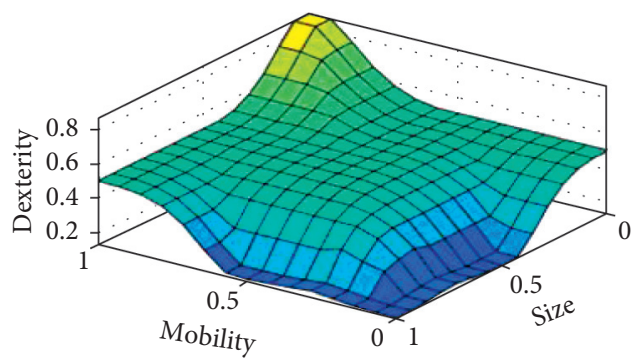

Figure 26: Fuzzy output surface for model M5.

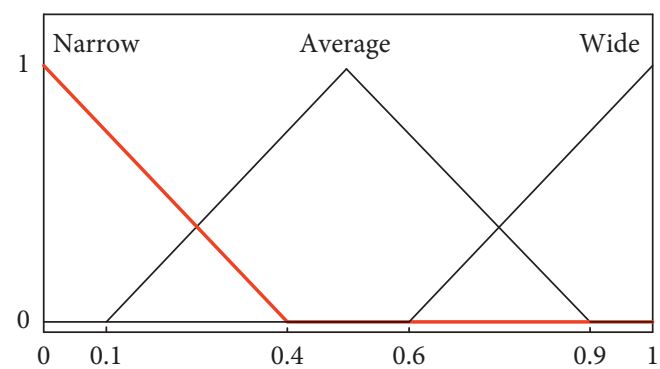

FIGURE 27: FV membership functions for LV "control range".

solution surfaces and modeling results obtained on their basis, are presented below.

Experimental studies of the method of formation of quantitative indicators of complexity of the local region of the environment were conducted in several test scenarios. Let us consider each of them. As shown in Figure 29, the group consists of three AMRs and five obstacles; positions of which are described in Table 1 . The CVS was modeled by lidar with a distance matrix of $16 \times 8$, a viewing angle of $90^{\circ}$

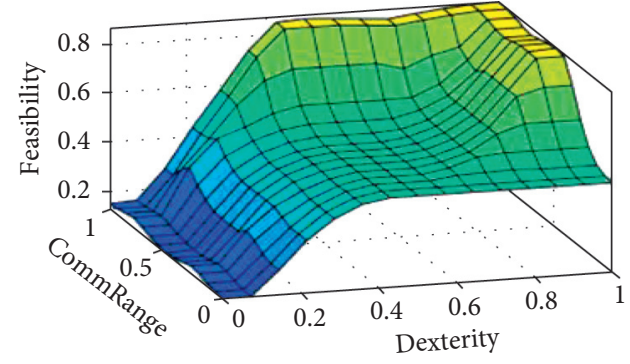

Figure 28: Fuzzy output surface for model M6.

horizontally and $45^{\circ}$ vertically, and a range of $20 \mathrm{~m}$. Figure 30 illustrates the lidar scanning of the environment by the respective robots.

The coordinates of the start and end moving points of AMR are given in Table 2 and Table 3. Figure 31 presents the results of simulating the movement of a group of robots using fuzzy modes [23-26]. Figures 31(a) to 31(d) show the results of simulating the movement of a group of AMR when reaching the endpoint from different angles.

As we can see from Figure 31, the environment looks most complex from the point of view of the AMR no. 1 (solid line), but its low value (less than 0.25 ) allowed all the robots to pass the group of obstacles as one formation, without collisions. The complexity estimations of AMR no. 2 and AMR no. 3 are somewhat similar because they have symmetrical positions in the group. Case 2: each robot in the group has its own complexity. The initial positions of the obstacles and the AMR group are shown in Figure 32.

The coordinates of the start and end moving points of the AMR group are given in Table 4 and Table 5 . As we can see in Figure 32, the group of AMR needs to pass an obstacle that is presented as a wall of spheres with singular radius and blocks $50 \%$ of the movement zone. Case 3 simulation results are shown in Figure 33.

As we can see from Figure 33, the different members of the AMR group have significantly different CVS views, which influence the process of motion and the assessment of the complexity of the environment. Figure 34 shows Case 2 stimulation results. In this case, the environment looks the most complex from the point of view of the robot no. 2 (dashed line), which was the reason for not achieving the endpoint on time. AMR no. 1 (solid line) assessed the complexity of the environment as 


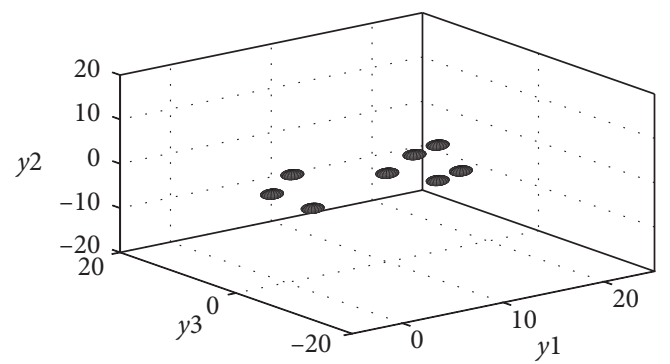

(a)

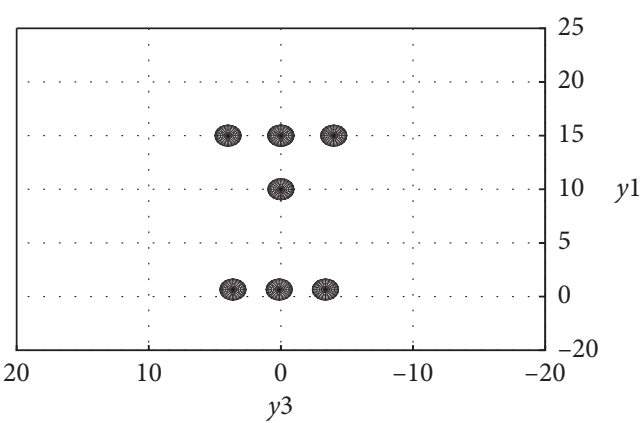

(b)

FIGURE 29: Initial positions of the obstacles and the group of AMR: (a) 3D view; (b) plan view.

TABLE 1: Obstacle coordinates.

\begin{tabular}{lccc}
\hline No. of obstacles & $y_{p 1}$ & $y_{p 3}$ & \\
\hline 1 & 10 & 0 & 0 \\
2 & 15 & -4 & 0 \\
3 & 15 & 4 & 0 \\
4 & 15 & 0 & 4 \\
5 & 15 & 0 & -4 \\
\hline
\end{tabular}

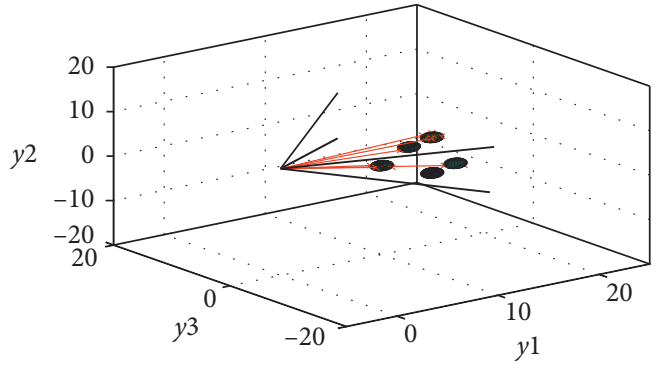

(a)

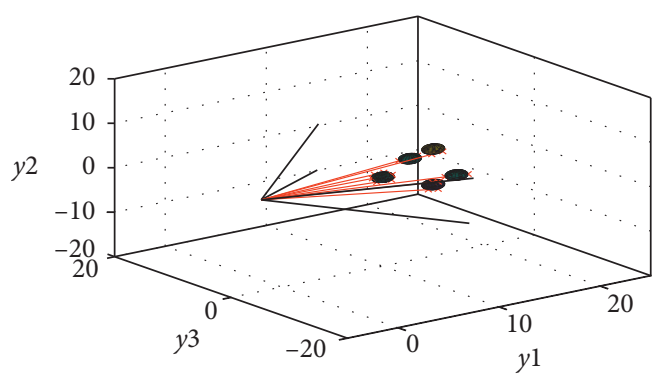

(c)

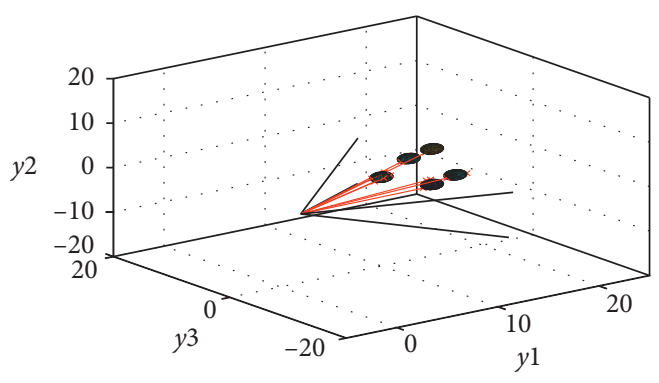

(e)

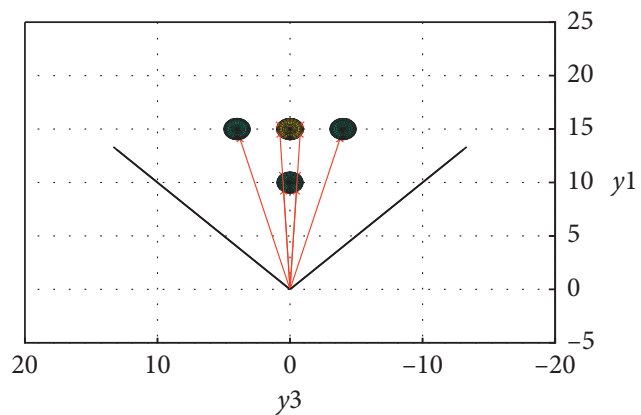

(b)

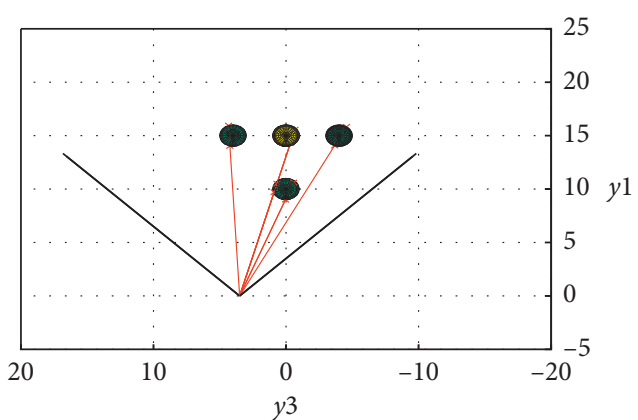

(d)

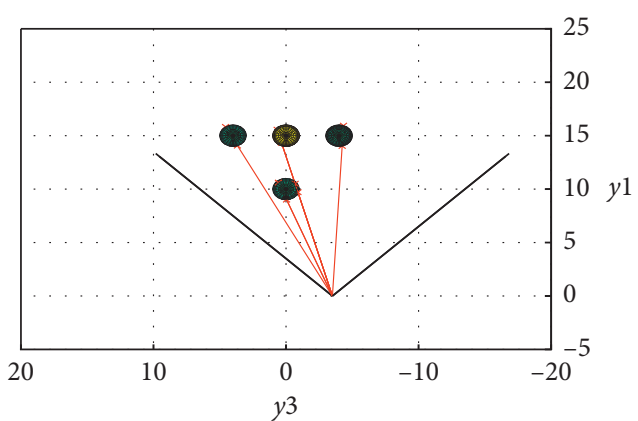

(f)

FIGURE 30: Simulating the work of CVS: (a) 3D view of AMR no. 1; (b) plan view of AMR no. 1; (c) 3D view of AMR no. 2; (d) plan view of AMR no. 2; (e) 3D view of AMR no. 3; (f) plan view of AMR no. 3. 
TABLE 2: Starting point coordinates.

\begin{tabular}{lccc}
\hline No. of the AMR & $y_{1}$ & $y_{3}$ & \\
\hline 1 (center) & 0 & 0 & 4 \\
2 (left) & 0 & 3.5 & -2 \\
3 (right) & 0 & -3.5 & -2 \\
\hline
\end{tabular}

TABLE 3: Endpoint coordinates.

\begin{tabular}{lccc}
\hline No. of the AMR & $y_{1}$ & $y_{3}$ & \\
\hline 1 (center) & 20 & 0 & $y_{2}$ \\
2 (left) & 20 & 3.5 & 4 \\
3 (right) & 20 & -3.5 & -2 \\
\hline
\end{tabular}

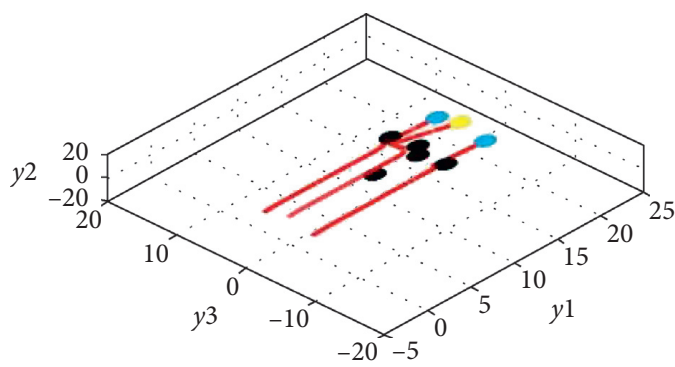

(a)

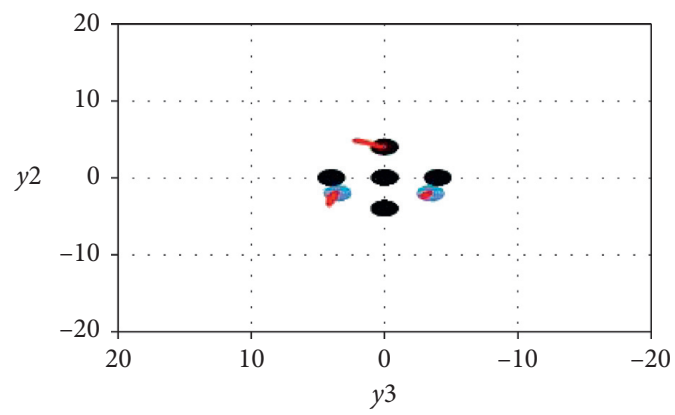

(c)

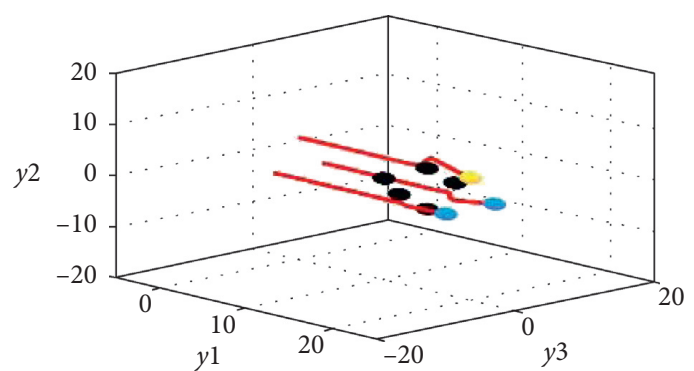

(b)

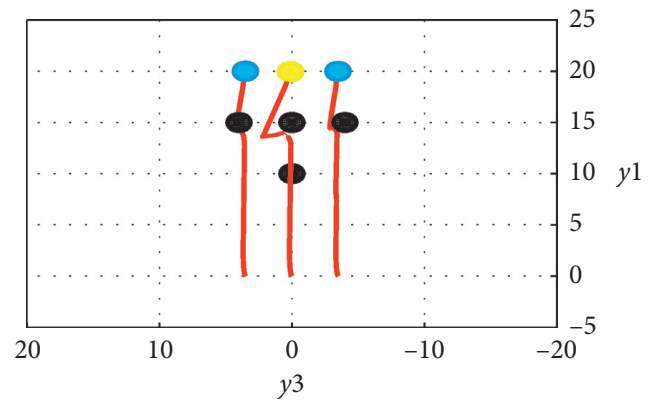

(d)

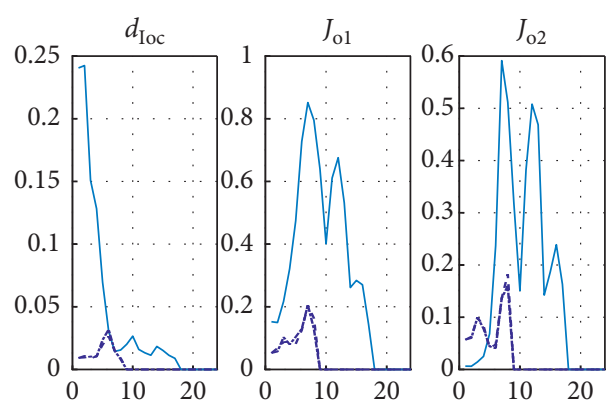

(e)

FIgURE 31: Case 1 simulation results. (a-d) Results of simulating the movement of a group of AMR when reaching the endpoint; (e) estimation of the complexity of the environment during movement.

rather complicated, but after left maneuvering indicators JYO1 and JYO2 began to decrease and the estimation changed to noncomplicated. The estimation of environment complexity by the AMR no. 3 (chain-dotted line) initially was the lowest one, and there was no need for motion correction during the simulation.

\section{Conclusions}

Conducted studies and experiments showed that the method of formation of estimations of local regions of the environment using the CVS data allows us to get an idea of how loaded they are with various objects, such as obstacles. Such 


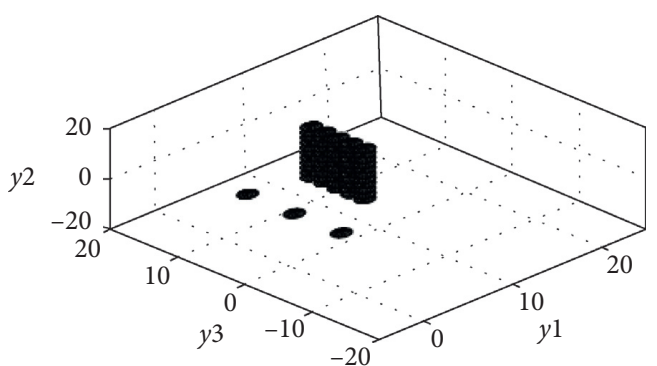

(a)

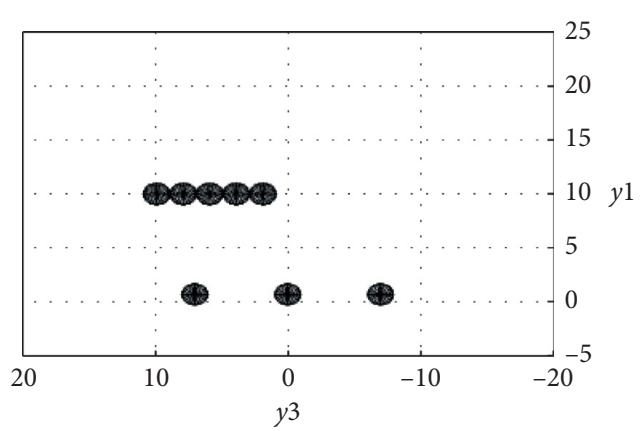

(b)

FIgURE 32: Initial positions of the obstacles and the group of AMR: (a) 3D view; (b) plan view.

TABLE 4: Starting point coordinates.

\begin{tabular}{lccc}
\hline No. of the AMR & $y_{1}$ & $y_{3}$ & \\
\hline 1 (center) & 0 & 0 & $y_{2}$ \\
2 (left) & 0 & 3 & 0 \\
3 (right) & 0 & -3 & 0 \\
\hline
\end{tabular}

TABLE 5: Endpoint coordinates.

\begin{tabular}{lccc}
\hline No. of the AMR & $y_{1}$ & $y_{3}$ & \\
\hline 1 (center) & 20 & 0 & 0 \\
2 (left) & 20 & 3 & 0 \\
3 (right) & 20 & -3 & 0 \\
\hline
\end{tabular}

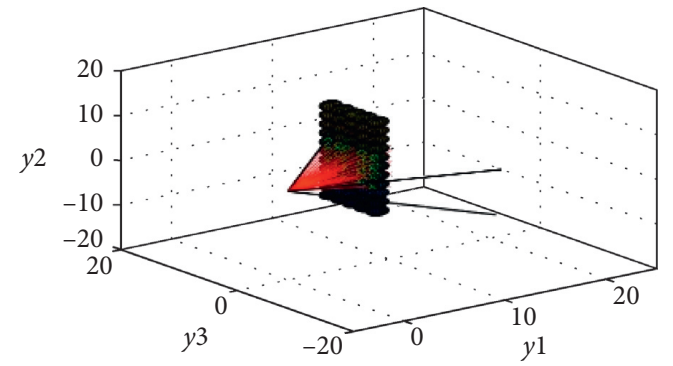

(a)

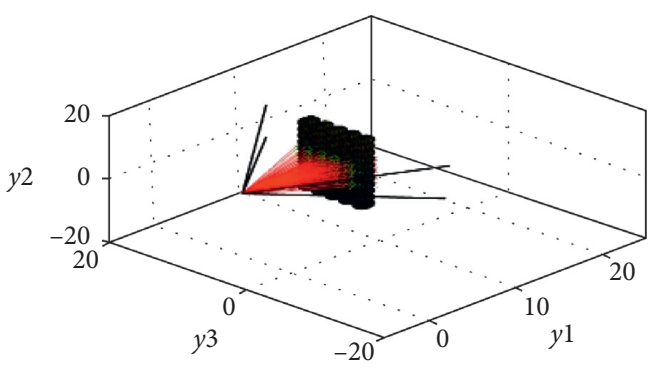

(c)

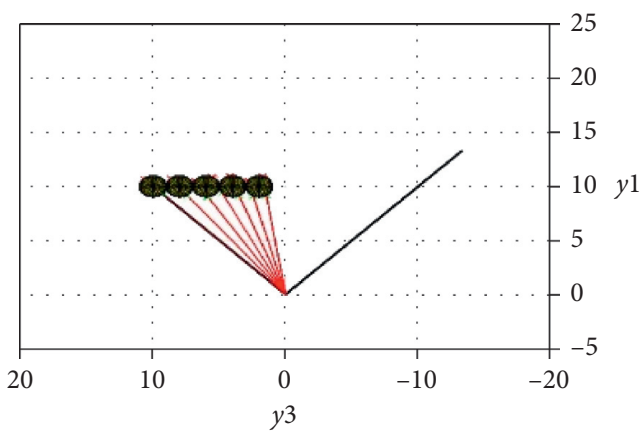

(b)

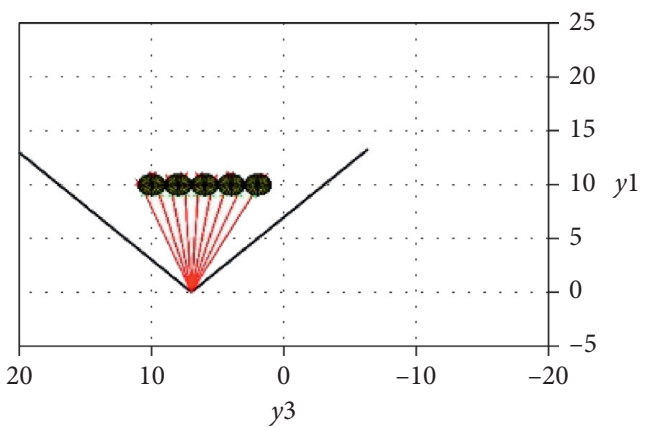

(d)

Figure 33: Continued. 


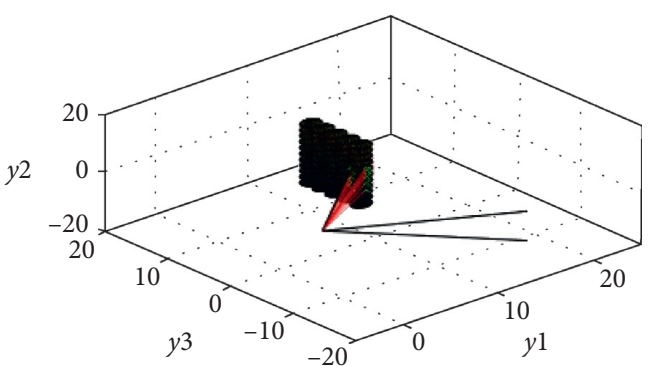

(e)

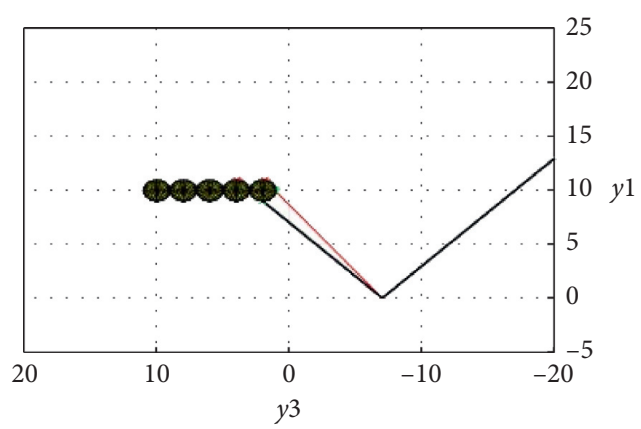

(f)

FIGURE 33: Simulating the work of CVS: (a) 3D view of AMR no. 1; (b) plan view of AMR no. 1; (c) 3D view of AMR no. 2; (d) plan view of AMR no. 2; (e) 3D view of AMR no. 3; (f) plan view of AMR no. 3.

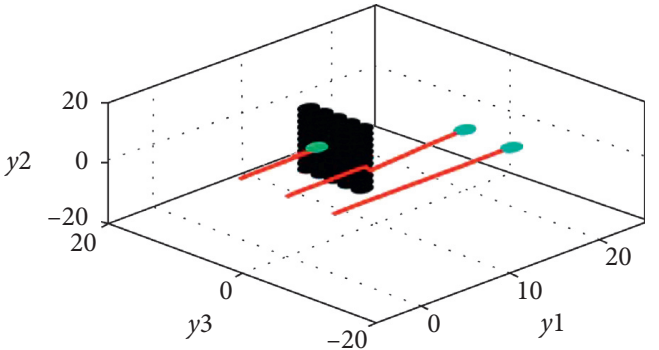

(a)

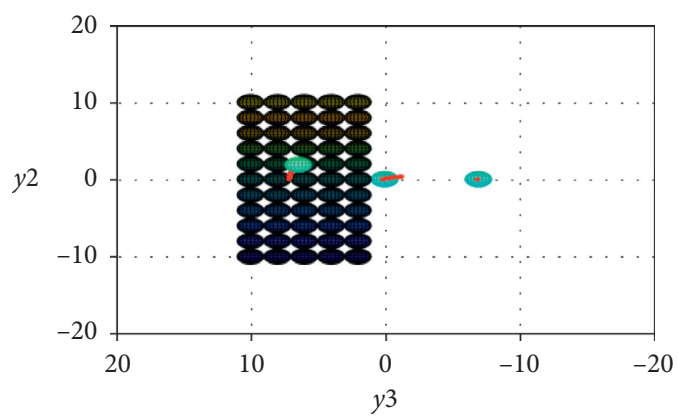

(c)

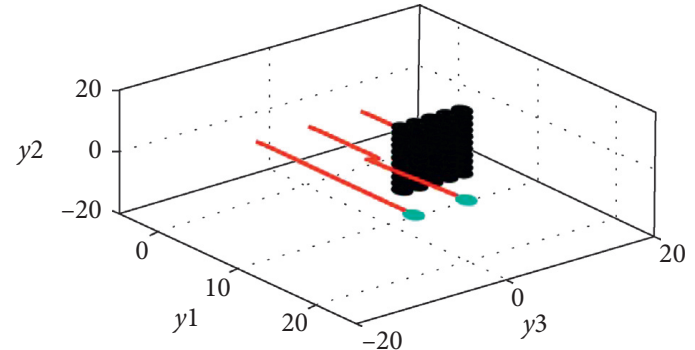

(b)

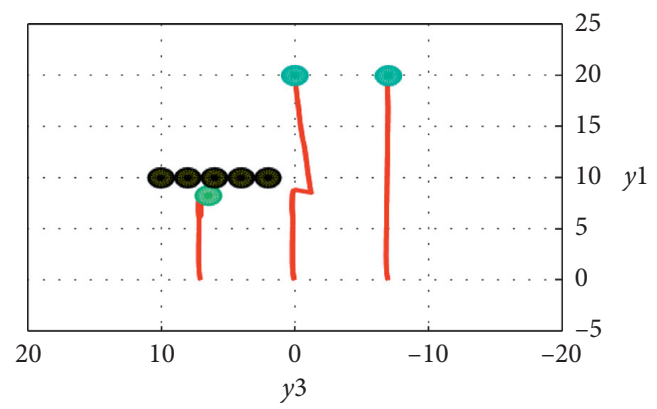

(d)
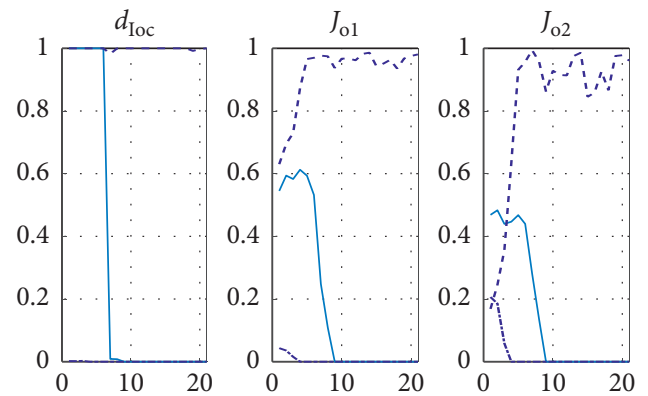

(e)

Figure 34: Case 1 simulation results. (a-d) Results of simulating the movement of a group of AMR when reaching the endpoint; (e) estimation of the complexity of the environment during movement.

data can be used for determining of optimal planning algorithm that meets the requirements of the current situation in terms of the cost of computing resources and the efficiency of the resulting trajectories [23-26]. If the considered local region has relatively small complexity, the planning task can be successfully solved based on such methods as virtual fields and fuzzy behavioral approach.

If the complexity is high, alternative approaches should be used, for example, the method of unstable modes. Data about the complexity of the environment can also be used for 
defying some of its regions as insurmountable for a single robot or a group of robots. The reasons for this decision may be a violation of security requirements, inability to keep the group formation, or completion of the task on time. The method of formation of a complex assessment of AMRs' characteristics allows obtaining quantitative estimations of their fitness for achieving the set objectives. These data are necessary for effective target distribution, reconfiguration of the group formation in case of changes in its structure, objective change, etc.

\section{Data Availability}

No data were used to support this study.

\section{Conflicts of Interest}

The authors declare that there are no conflicts of interest.

\section{Authors' Contributions}

All authors contributed to the experiments and writing of this paper.

\section{Acknowledgments}

This work was conducted at the Scientific Design Bureau of Robotics and Control Systems (SDBRCS) in Taganrog, Russia, and funded by the Russian Foundation for Basic Research (RFBR) (grant no. 18-19-00621).

\section{References}

[1] E. Masehian, M. Jannati, and T. Hekmatfar, "Cooperative mapping of unknown environments by multiple heterogeneous mobile robots with limited sensing," Robotics and Autonomous Systems, vol. 87, pp. 188-218, 2017.

[2] M. Gadd and P. Newman, "Checkout my map: version control for fleetwide visual localisation," in Proceedings of the 2016 IEEE/RSJ International Conference on Intelligent Robots and Systems (IROS), pp. 5729-5736, Daejeon, South Korea, October 2016.

[3] L. Contreras, O. Kermorgant, and P. Martinet, "Efficient decentralized collaborative mapping for outdoor environments," in Proceedings of the 2018 Second IEEE International Conference on Robotic Computing, Laguna Hills, CA, USA, January 2018.

[4] T. Andre, D. Neuhold, and C. Bettstetter, "Coordinated multirobot exploration: out of the box packages for ROS," in Proceedings of the 2014 IEEE Globecom Workshops (GC Wkshps), Austin, TX, USA, December 2014.

[5] F. Peter, Bloesch Michael, K. Philipp et al., "Collaborative navigation for flying and walking robots," in Proceedings of the 2016 IEEE/RSJ International Conference on Intelligent Robots and Systems (IROS), pp. 2859-2866, Daejeon, South Korea, October 2016.

[6] C. Titus, L. Simon, M. Dymczyk, S. Magnenat, and S. Roland, "Map API-scalable decentralized map building for robots," in Proceedings of the 2015 IEEE International Conference on Robotics and Automation (ICRA), pp. 6241-6247, Seattle, WA, USA, May 2015.
[7] D. M. Lyons, R. C. Arkin, S. Jiang, M. O’Brien, F. Tang, and P. Tang, "Performance verification for robot missions in uncertain environments," Robotics and Autonomous Systems, vol. 98, pp. 89-104, 2017.

[8] D. M. Lyons, A. Ronald, T.-M. Liu, S. Jiang, and P. Nirmal, "Verifying performance for autonomous, robot missions with uncertainty," IFAC Proceedings Volumes, vol. 46, no. 10, pp. 179-186, 2013, http://fordham.bepress.com/frcv_ facultypubs $/ 1$.

[9] F. Kamil, T. S. Hong, W. Khaksar, M. Y. Moghrabiah, N. Zulkifli, and S. A. Ahmad, "New robot navigation algorithm for arbitrary unknown dynamic environments based on future prediction and priority behavior," Expert Systems With Applications, vol. 86, pp. 274-291, 2017.

[10] Z. Wu and F. Lin, "Obstacle prediction-based dynamic path planning for a mobile robot," International Journal of Advancements in Computing Technology (IJACT), vol. 4, no. 3, 2012.

[11] Z. Y. Ibrahim, A. T. Rashid, and A. F. Marhoon, "Predictionbased path planning with obstacle avoidance in dynamic target environment," Basrah Journal for Engineering Sciences, vol. 16, no. 2, 2016.

[12] M. Nazarahari, E. Khanmirza, and S. Doostie, "Multi-objective multi-robot path planning in continuous environment using an enhanced genetic algorithm," Expert Systems With Applications, vol. 115, pp. 106-120, 2019.

[13] P. Shamsinejad, M. Saraee, and F. Sheikholeslam, "A new path planner for autonomous mobile robots based on genetic algorithm," in Proceedings of the 2010 3rd International Conference on Computer Science and Information Technology, Chengdu, China, July 2010.

[14] C. Lamini, S. Benhlima, and A. Elbekri, "Genetic algorithm based approach for autonomous mobile robot path planning," Procedia Computer Science, vol. 127, pp. 180-189, 2018.

[15] X. Zhang, Y. Zhao, N. Deng, and K. Guo, "Dynamic path planning algorithm for a mobile robot based on visible space and an improved genetic algorithm," International Journal of Advanced Robotic Systems, vol. 13, no. 3, p. 91, 2016.

[16] H. Guo, Z. Mao, W. Ding, and P. Liu, "Optimal search path planning for unmanned surface vehicle based on an improved genetic algorithm," Computers \& Electrical Engineering, vol. 79, p. 106467, 2019.

[17] A. Bakdi, A. Hentout, H. Boutami, A. Maoudj, O. Hachour, and B. Bouzouia, "Optimal path planning and execution for mobile robots using genetic algorithm and adaptive fuzzylogic control," Robotics and Autonomous Systems, vol. 89, pp. 95-109, 2017.

[18] J. Liu, B.-Y. Ma, N. Fry et al., "Exploration robots for harsh environments and safety," IFAC-PapersOnLine, vol. 48, no. 10, pp. 41-45, 2015.

[19] L. Pfotzer, S. Klemm, A. Roennau, J. M. Zöllner, and R. Dillmann, "Autonomous navigation for reconfigurable snake-like robots in challenging, unknown environments," Robotics and Autonomous Systems, vol. 89, pp. 123-135, 2017.

[20] C. M. Gifford, R. Webb, J. Bley et al., "A novel low-cost, limited-resource approach to autonomous multi-robot exploration and mapping," Robotics and Autonomous Systems, vol. 58, no. 2, pp. 186-202, 2010.

[21] S. Shao, Yu Peng, C. He, and Y. Du, "Efficient path planning for UAV formation via comprehensively improved particle swarmoptimization," ISA Transactions, vol. 97, pp. 415-430, 2020. 
[22] E. S. Low, P. Ong, and K. C. Cheah, "Solving the optimal path planning of a mobile robot using improved Q-learning," Robotics and Autonomous Systems, vol. 115, pp. 143-161, 2019.

[23] D. A. Beloglazov, V. F. Guzik, E. Y. Kosenko et al., Intelligent Planning of Vehicles Path in the Environment with Obstacles, V. K. Pshikhopov, Ed., Fizmatlit, Moscow, Russia, 2014.

[24] D. A. Beloglazov, A. R. Gaiduk, E. Y. Kosenko et al., Group Management of Moving Objects in Uncertain Environments, V. H. Pshihopov, Ed., Fizmatlit, Moscow, Russia, 2015.

[25] V. Pshikhopov, M. Medvedev, V. Shibanov, and I. O. Shapovalov, "Path planning and control of vehicles in 3D environment using unstable modes," in Proceedings of the 2017 4th International Conference on Control, Decision and Information Technologies (CoDIT'17), pp. 621-626, Barcelona, Spain, April 2017.

[26] E. Kosenko, D. Beloglazov, and V. Finaev, "Vehicles fuzzy control under the conditions of uncertainty," in Path Planning for Vehicles Operating in Uncertain 2D Environments, V. Pshikhopov, Ed., pp. 97-136, Elsevier, Amsterdam, Netherlands, 2017. 